\title{
Innovación e improvisación en el marco de la pandemia de COVID-19: relato de una experiencia
}

\author{
Innovation and improvisation in the framework of the COVID-19 \\ pandemic: an experience report
}

\author{
Laura Sombra Del Río \\ https://orcid.org/0000-0002-7800-631X \\ laura.delrio@ing.unlp.edu.ar \\ UIDET IMApEC - Depto. Ciencias Básicas, \\ Facultad de Ingeniería | UNLP| Argentina
}

\section{Eugenia Boero}

https://orcid.org/0000-0003-4921-3077

eugeniaboero@outlook.es

Cátedra de Matemática A - Depto. Ciencias

Básicas, Facultad de Ingeniería | UNLP | Argentina

\author{
Patricia Knopoff \\ https://orcid.org/0000-0001-8596-0198 \\ patricia.knopoff@ing.unlp.edu.ar \\ Cátedra de Matemática A - Depto. Ciencias Básicas, \\ Facultad de Ingenieria | UNLP |Argentina
}

\author{
Leonardo Ciliberti \\ https://orcid.org/0000-0002-0727-8422 \\ leocili@gmail.com \\ Cátedra de Matemática A - Depto. Ciencias Básicas, \\ Facultad de Ingeniería | UNLP |Argentina
}

\section{RESUMEN}

En el presente trabajo se relata la experiencia de nuestro equipo docente en el marco de la contingencia provocada por la pandemia de COVID-19. Nuestra universidad decidió en este particular contexto ratificar su calendario académico y garantizar la continuidad pedagógica a través de la modalidad de enseñanza a distancia. Este requerimiento nos puso a los docentes de la casa a pensar y a actuar con gran celeridad, seleccionando las herramientas y metodologías que consideramos más adecuadas para garantizar el derecho a continuar estudiando a la mayoría de nuestros estudiantes. En este trabajo, queremos presentar las estrategias didácticas llevadas a cabo por nuestro equipo docente -el cual está a cargo de una comisión de la cátedra Matemática A, de la Facultad de Ingeniería de la Universidad Nacional de La Plata-, algunas de las dificultades que nos hemos encontrado en este camino y algunos resultados que nos permiten valorar positivamente la experiencia.

\section{ABSTRACT}

In this paper, the experience of our teaching team in the context of the contingency caused by the COVID-19 pandemic is reported. Our university decided in this particular context to ratify its academic calendar and guarantee pedagogical continuity through the distance learning modality. This requirement put the teachers of the house to think and act with great speed, selecting the tools and methodologies that we consider most appropriate to guarantee the right to continue studying the majority of our students. In this work, we want to present the didactic strategies carried out by our teaching team - which is in charge of a committee of the Mathematical Chair A, of the Faculty of Engineering of the National University of La Plata -, some of the difficulties that We have found ourselves on this path and some results that allow us to positively value the experience.

PALABRAS CLAVE Educación virtual de emergencia, COVID-19, Enseñanza de la Matemática, TIC y Educación
KEY WORDS

Virtual emergency education, COVID-19,

Teaching of Mathematics, ICT and Education 


\section{PRESENTACIÓN}

La declaración de la pandemia de COVID-19 y el comienzo de casos positivos en nuestro país desembocaron en acciones del gobierno nacional. La más importante de ellas fue el aislamiento social obligatorio que transformó la cotidianeidad de todos los argentinos en diversos formatos.

En el ámbito universitario y considerando la autarquía propia de las casas de altos estudios, hubieron diferentes posiciones. A la par que la Universidad de Buenos Aires reprogramaba su calendario académico, posponiendo el inicio de clases para el mes de junio (Cafferata, 2020), otras universidades se deciden por la continuidad (Fernández, 2020) y entre ellas, la Universidad Nacional de La Plata ratifica su calendario y comunica a sus docentes sobre la necesidad de mantener la continuidad pedagógica en todas sus Facultades (UNLP, 2020).

Ante este escenario, lo primero que discernimos fueron las diferencias existentes en los modos de enseñanza presenciales y a distancia. Estábamos ingresando a esta segunda situación y entendíamos que debíamos distinguir entre lo que se establece habitualmente para impartir cursos de enseñanza a distancia normales, es decir previstos y planificados en ese contexto, con el inicio de este escenario de enseñanza virtual de emergencia que nos imponía el aislamiento obligatorio.

Ante este escenario, lo primero que discernimos fueron las diferencias existentes en los modos de enseñanza presenciales y a distancia. Estábamos ingresando a esta segunda situación y entendíamos que debíamos distinguir entre lo que se establece habitualmente para impartir cursos de enseñanza a distancia normales, es decir previstos y planificados en ese contexto, con el inicio de este escenario de enseñanza virtual de emergencia que nos imponía el aislamiento obligatorio.

Probablemente la palabra más adecuada para esos días de inicio sea improvisación, pero no en el sentido peyorativo de hacer algo "a los ponchazos", como salga, sin planificación y sin objetivos claros. Entendemos 
que hemos transitado el camino de la improvisación en el sentido más profundo que se le da en el teatro: "Un buen actor de improvisación teatral debe estar atento, saber escuchar, estar abierto a aceptar propuestas, así como a responder de forma divertida y clara en base a la historia que se está transmitiendo" (entradas.com, 2019).

Es en ese sentido, es que nos abocamos a hacer lo mejor que se podía con todo lo que se tenía, ante una situación desconocida, con objetivos claros y planificando -tal vez no tan a largo plazo- cada paso que dábamos.

Así, hemos logrado transitar la primera mitad de nuestra asignatura -incluyendo la primera evaluación- en situación de completa virtualidad y que relatamos a continuación.

\section{DESCRIPCIÓN DE LA EXPERIENCIA}

El domingo 15 de marzo de 2020, el Presidente de la Nación anunciaba en conferencia de prensa la suspensión de clases presenciales en los niveles inicial, primario, secundario y superior, en todo el territorio de la República Argentina, convocando a los docentes a garantizar la continuidad pedagógica a través de enseñanza remota. Casi en simultáneo, la Universidad Nacional de La Plata (UNLP) hacía lo propio en el marco de la institución. Ese mismo día, mientras se sucedían los anuncios, comenzamos como equipo docente a pensar cómo, con qué estrategias y con qué herramientas, haríamos frente a este nuevo desafío.

\section{El domingo 15 de marzo de 2020, el Presidente de la Nación anunciaba en conferencia de prensa la suspensión de clases presenciales en los niveles inicial, primario, secundario y su- perior, en todo el territorio de la República Argentina, convo- cando a los docentes a garantizar la continuidad pedagógica a través de enseñanza remota.}

Para ordenar este relato, dedicaremos primero algunas líneas a describir cómo veníamos funcionando en nuestra comisión de la Cátedra de Matemática A de la Facultad de Ingeniería de la UNLP durante la presencialidad. Nuestros estudiantes concurren a clase tres veces por semana y cada clase dura cuatro horas. Durante la misma, los docentes acompañamos a los estudiantes en su proceso de aprendizaje con distintas estrategias. Realizamos una presentación del tema del día utilizando el pizarrón y el proyector. De esta presentación, participan activamente los estudiantes, quienes han leído previamente el material impreso que proporciona la Cátedra. Durante el resto de la clase (la mayor parte de la mis$\mathrm{ma})$, los estudiantes trabajan en equipos siguiendo el material impreso y los docentes (profesora, jefa de trabajos prácticos y los dos ayudantes) recorremos el aula asistiendo a los estudiantes, discutiendo con ellos en los grupos, respondiendo dudas, etc. (Búcari, Abate y Melgarejo, 2004). 
También contamos con un espacio virtual, un aula en Google Classroom, que utilizamos para enviar información, actividades adicionales, recursos educativos digitales interactivos (principalmente desarrollados con el software libre GeoGebra), para complementar lo trabajado en el aula convencional.

En este marco, ese día domingo resolvimos aprovechar este espacio virtual, que funcionaba desde principios de marzo, para llevar a cabo el nuevo desafío de enseñar en forma completamente virtual una asignatura que fue concebida para la presencialidad, y sin contar con tiempo físico para repensarla en el nuevo entorno.

Lo primero que hicimos entre el domingo y el lunes por la mañana, fue realizar un video (https://youtu.be/dV4tt5dl9Gs) explicando a los estudiantes la situación de la pandemia del COVID-19, utilizando las herramientas matemáticas que estudiamos en la materia, y dándoles la tranquilidad de que allí estaríamos sus docentes para ayudarlos y acompañarlos y para garantizar su derecho al estudio. Les indicamos también cómo sería la modalidad de trabajo mientras durara el aislamiento social preventivo y obligatorio. Nos pareció sumamente importante hacer esto ese mismo día, de modo de tener funcionando un sistema, que podría ir perfeccionándose en el tiempo, desde el día 0.

\section{Lo primero que hicimos entre el domingo y el lunes por la ma- ñana, fue realizar un video (https://youtu.be/dV4tt5dI9Gs) explicando a los estudiantes la situación de la pandemia del COVID-19, utilizando las herramientas matemáticas que estu- diamos en la materia, y dándoles la tranquilidad de que allí es- taríamos sus docentes para ayudarlos y acompañarlos y para garantizar su derecho al estudio.}

Junto con el video, subimos algunas autoevaluaciones que habíamos desarrollado con Google Forms el año anterior, sobre los temas que habíamos comenzado a tratar mientras pudimos concurrir al aula convencional.

De esta manera, el día lunes, en el horario de cursada habitual, nuestros estudiantes tenían trabajo por hacer: mirar el video y realizar las primeras autoevaluaciones, mientras el equipo docente se terminaba de re-organizar y probar herramientas nuevas para poder realizar videoconferencias.

Las herramientas y estrategias que finalmente consensuamos utilizar para llevar a cabo esta tarea fueron las siguientes:

- Utilización de un sistema de videoconferencia para las discusiones grupales iniciales: en primer lugar, utilizamos Zoom y luego, al conocerse públicamente las problemáticas de seguridad que esta aplicación tenía (Magnani, 2020), pasamos a Webex de Cisco. Durante estas comu- 
nicaciones utilizamos pizarras virtuales (Jamboard y Microsoft Whiteboard) y el software libre GeoGebra (www.geogebra.org). Una cuestión fundamental, como se mostrará más adelante, fue la decisión de grabar estos encuentros para dejar a disposición de los estudiantes que, por diversas razones, no podían participar en vivo y en directo de los mismos.

- Para las consultas, dividimos a los estudiantes en grupos, quedando cada uno asistido por un integrante del equipo docente. Estas consultas se llevan a cabo en dos modalidades: por videoconferencia y por escrito a través de Google Classroom. La segunda opción atiende a que uno de los docentes no cuenta con equipo informático adecuado para realizar consultas por videoconferencia, así como tampoco algunos de los estudiantes. Además, este espacio resulta muy bien aprovechado por los alumnos que, habiendo realizado alguna actividad, desean o necesitan que nosotros las revisemos y se les dificulta enseñárnosla mediante videoconferencia. En estos casos, ellos suben fotos de la actividad sobre la cual quieren consultar y este docente les hace una devolución.

- También continuamos trabajando con autoevaluaciones, que sirven a los estudiantes para monitorear su propio progreso y nos sirven a los docentes para conocer qué temáticas necesitamos revisitar para que queden más claras.

- Por último, compartimos con los estudiantes recursos realizados en GeoGebra para favorecer la visualización de conceptos matemáticos y la exploración de los mismos, tal como lo hacíamos como complemento en la presencialidad (Del Río, 2017, Del Río, 2018) y modificamos algunos incluyendo consignas en forma de preguntas para que puedan responder y así nosotros también tener una retroalimentación sobre los que ellos están comprendiendo sobre esos recursos.

\section{LA VOZ DE NUESTROS ESTUDIANTES}

En este apartado queremos compartir los resultados de una encuesta anónima que realizamos a nuestros estudiantes, donde ellos tuvieron la posibilidad de expresarse y ayudarnos a mejorar esta experiencia. La misma fue respondida por 33 alumnos (en las clases virtuales en vivo participan alrededor de 45 alumnos y en las presenciales asistían poco más de 60).

En la encuesta, solicitamos que valoren en una escala de 1 a 5 cada uno de los recursos que pusimos a su disposición para conocer su opinión, que nos realicen sugerencias sobre otros recursos que ellos consideren que podrían resultar de utilidad y que propongan otras formas de organización que pudieran ser mejores a las que nosotros propusimos.

En primer lugar, queremos compartir los resultados de la pregunta más general que contenía la encuesta: “QQué te parece, en general, el modo en el que organizamos la cursada para este período de clases virtuales?" Como se puede ver en el gráfico de la Figura 1, la percepción de los estudiantes sobre el desempeño del equipo docente es más que positivo.

Trayectorias Universitarias | Vol. 6 - № 10 | 2020 | ISSN 2469-0090 


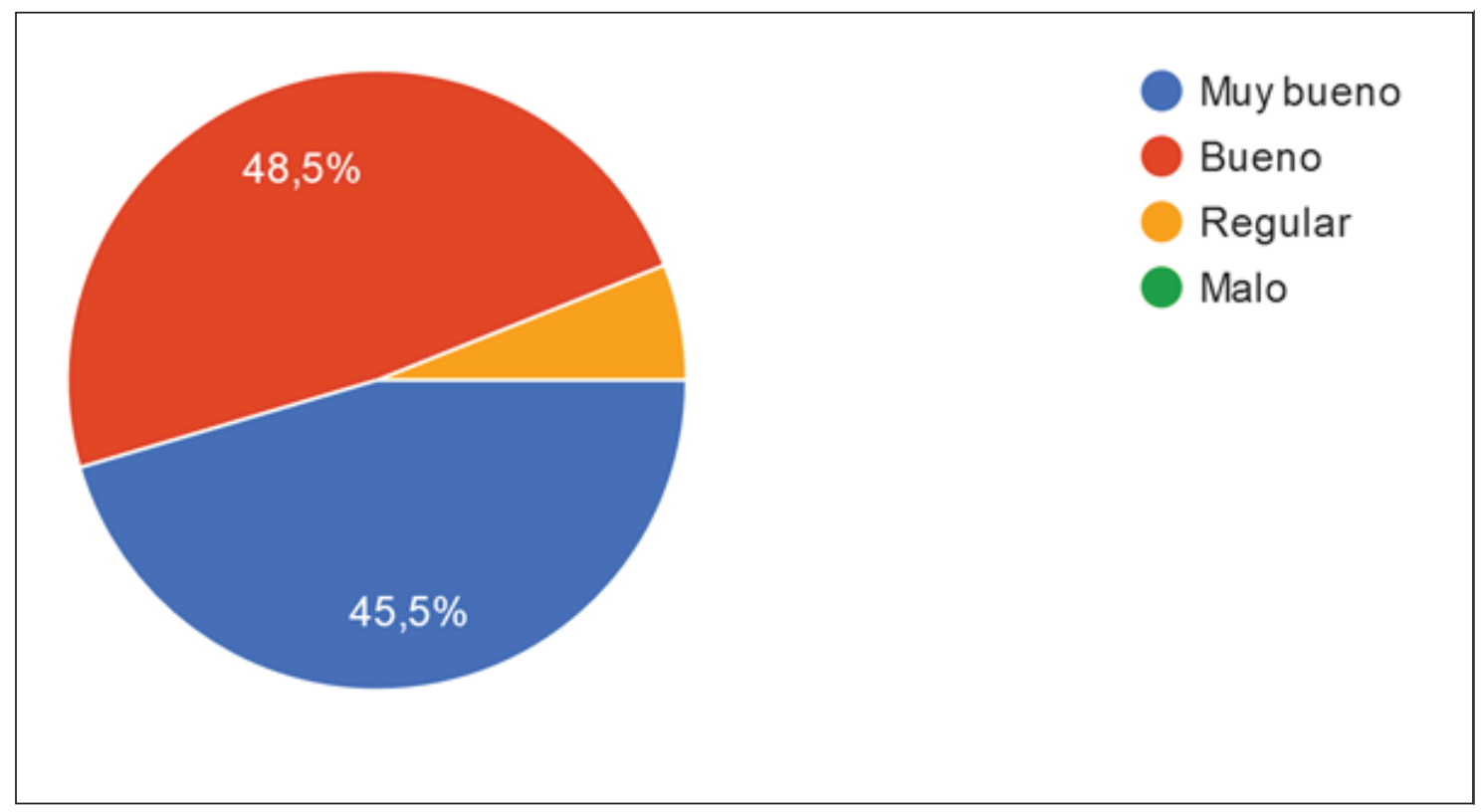

Figura 1 - Respuestas de los estudiantes a la pregunta ¿Qué te parece, en general, el modo en el que organizamos la cursada para este período de clases virtuales?

Respecto de la valoración que hacen de cada uno de los distintos recursos que pusimos a su disposición, pueden verse los resultados en la Figura 2.
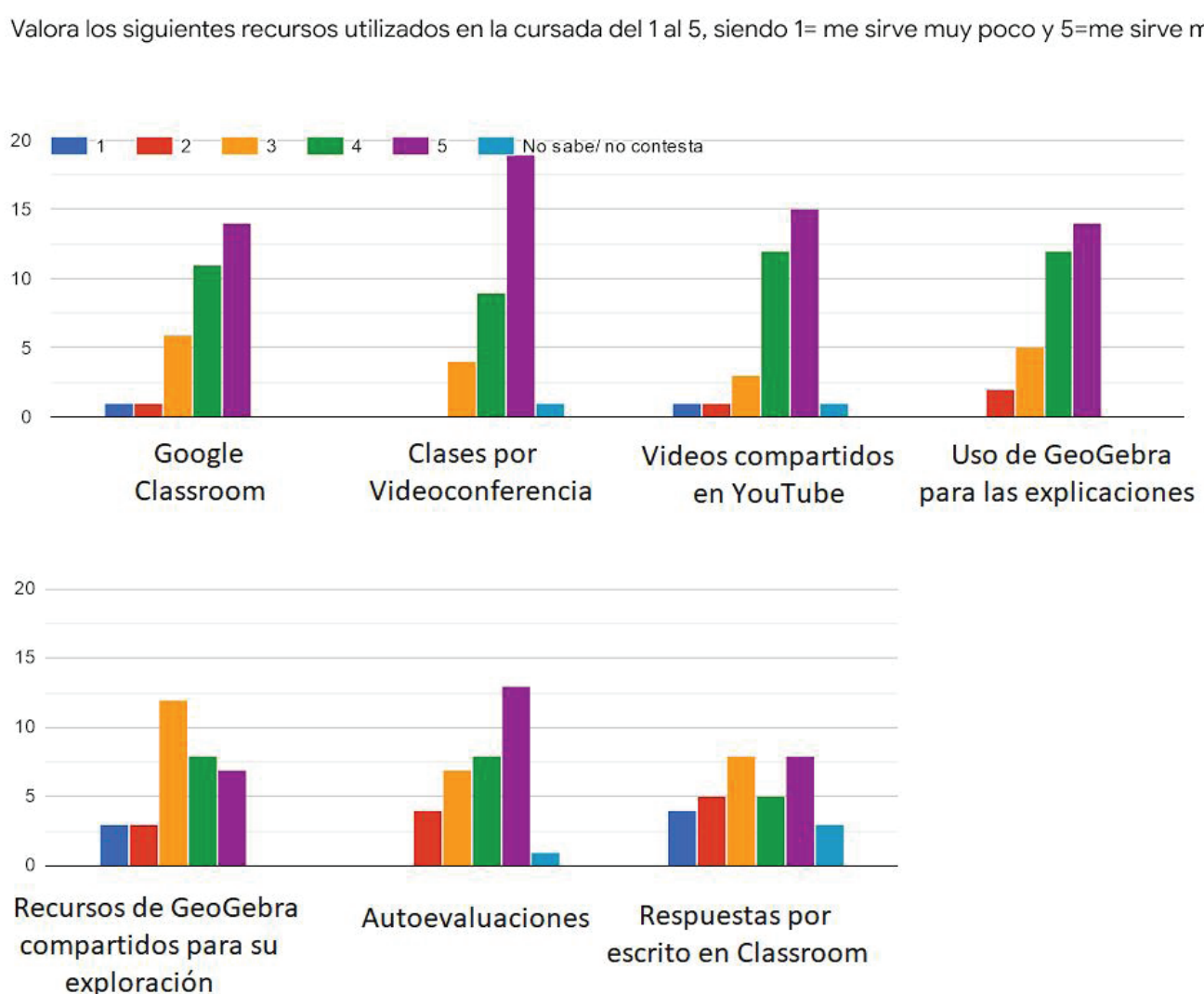

Figura 2 - Valoración de los estudiantes de cada una de las herramientas utilizadas para la clase. 
De ese gráfico se desprende que todos los recursos fueron bien valorados, pero se observa una preferencia, por parte de los estudiantes, por aquellos recursos que requieren un rol más pasivo por su parte (preferencia del uso de GeoGebra acompañando las explicaciones por encima del uso autónomo por su parte; preferencia de las explicaciones en videoconferencia por encima de las enviadas por escrito, que requieren otro nivel de procesamiento por su parte). Lejos de ser esta interpretación una crítica a nuestros alumnos, entendemos que estas preferencias se vinculan con una cuestión cultural que es nuestro deber cuestionar junto a ellos para poder avanzar hacia una forma de trabajo más autónoma.

todos los recursos fueron bien valorados, pero se observa una preferencia, por parte de los estudiantes, por aquellos recursos que requieren un rol más pasivo por su parte (preferencia del uso de GeoGebra acompañando las explicaciones por encima del uso autónomo por su parte; preferencia de las explicaciones en videoconferencia por encima de las enviadas por escrito, que requieren otro nivel de procesamiento por su parte).

Otra de las preguntas de la encuesta se refería a qué otros recursos, más allá de los propuestos por los docentes, utilizan en el marco de esta contingencia. Muchos indicaron que recurren a canales de profesores Edutubers, grupos de WhatsApp en los que se contactan con sus propios compañeros o algunos organizados por las agrupaciones políticas estudiantiles, en los cuales tienen contacto con estudiantes de otras comisiones o incluso más avanzados en la carrera. Algunos mencionaron bibliografía y unos pocos consultan con familiares que tienen conocimientos sobre la materia. Que recurran más a Edutubers que a libros era una respuesta esperada por nosotros, y refuerza lo mencionado en el párrafo anterior. Vimos con agrado que se organicen por WhatsApp para apoyarse entre compañeros, ya que el trabajo en equipo es muy importante en el contexto de nuestra cátedra, siempre lo promovemos y era una de las cosas que más temíamos que se perdería en este contexto de contingencia.

Una de las preocupaciones más grandes que nos acompañó desde un comienzo fue la de los estudiantes que, por diversas razones, no pudieran seguirnos en las clases en vivo. En el aula presencial, teníamos entre 60 y 70 alumnos que acudían regularmente, mientras que en las sesiones por videoconferencia rondan los 45 . Si bien la disminución fue relativamente pequeña (o inferior a la que creíamos que tendríamos), esos pocos alumnos de los cuáles no teníamos noticias no dejaban de preocuparnos. Es por esto que les enviamos algunos correos electrónicos para animarlos a seguir estudiando, y convocamos a los alumnos activos a intentar contactar a sus compañeros para motivarlos también. 
En el aula presencial, teníamos entre 60 y 70 alumnos que acudían regularmente, mientras que en las sesiones por videoconferencia rondan los $\mathbf{4 5}$. Si bien la disminución fue relativamente pequeña (o inferior a la que creíamos que tendríamos), esos pocos alumnos de los cuáles no teníamos noticias no dejaban de preocuparnos. Es por esto que les enviamos algunos correos electrónicos para animarlos a seguir estudiando

Con el tiempo, algunos de estos estudiantes fueron reportándose por correo electrónico y sus mensajes nos señalaron que estábamos en buen camino. Por ejemplo, un estudiante que nos envió una consulta por mail y al presentarse nos contó que no nos seguía en vivo por su mala conectividad y que luego veía los videos en YouTube. Al preguntarle cómo venía con la materia, nos contó que estaba casi al día y que solo le faltaba ver los últimos dos videos disponibles. Otro estudiante nos contó que compartía una computadora con seis personas en su casa y que tenía que turnarse por lo que no podía estar siempre presente, pero también logró llevar la materia al día.

Si bien los integrantes del equipo poseíamos formaciones y experiencias diversas en relación al uso de las tecnologías digitales, una de las reflexiones en las que convergemos a la luz de estos resultados se vincula con el gran potencial que pueden tener estas herramientas que, sin lugar a dudas, nos impulsarán a utilizarlas más asiduamente y con nuevos sentidos cuando regresemos a la presencialidad.

\section{CONCLUSIONES}

Escribimos este trabajo con el deseo de que nuestra experiencia en este marco de enseñanza remota de emergencia no sea de utilidad para nadie, ya que quisiéramos no tener que atravesar nunca más por una situación similar a la actual. Sin embargo, todo indica que esto se prolongará en el tiempo $y$, además, nadie puede garantizar que en el futuro, cercano o lejano, no vuelva a pasar, por lo que tememos que sí puede resultar de utilidad.

Por otro lado, entendemos que nuestra respuesta ante la emergencia planteada es consecuencia de una suma de factores independientes entre sí, pero que de alguna manera se conjugaron simultáneamente: la estructura de nuestro equipo de trabajo, ya consolidado previamente; la capacidad personal de cada uno de nosotros para responder con rapidez a nuevos escenarios; y una cuota importante de buena voluntad y predisposición. Estas condiciones nos hacen pensar en una intransferibilidad de la experiencia, no solo a otros, sino a nosotros mismos en otra circunstancia.

Entendemos que en general la estrategia de compartir las experiencias en formato de trabajo académico tiene un gran componente de expectativa de transferibilidad. A pesar de lo expuesto previamente, 
no queremos perder la oportunidad de hacerlo ya que creemos que las estrategias y herramientas adoptadas han servido para garantizar el derecho a estudiar a una gran mayoría de nuestros estudiantes a cargo en este difícil y complejo escenario.

Es posible que la lectura de este trabajo pueda resultar inspiradora para alguien; para nosotros, escribir estas líneas nos hace navegar entre lo académico y lo épico. Relatar nuestra experiencia desde el marco mismo de la emergencia sanitaria, repensando el camino recorrido y mirándolo a la distancia como si hubiera ocurrido en otra época -aún si saber cuándo ni cómo se cerrará este episodio- no deja de tener un sesgo catártico y exorcizador. 


\section{BIBLIOGRAFIA}

Bucari, N., Abate, S. M. y Melgarejo, A. (2004). “Un cambio en la enseñanza de las matemáticas en las carreras de ingeniería de UNLP: propuesta, criterios y alcance". Cuarto Congreso Argentino de Enseñanza de la Ingeniería. Buenos Aires.

Cafferata, M. (5 de abril de 2020) “Las clases en la UBA comenzarán el $1^{\circ}$ de junio". Página 12. Recuperado de https://www.pagina12.com.ar/257588-lasclases-en-la-uba-comenzaran-el-1-de-junio

Del Río, L. (2017) !Visualization of limits of functions of two variables!. GeoGebra Global Gathering, Congreso llevado a cabo en Linz, Austria.

Del Río, L. (2018) ¡Learning Vector Calculus. How can GeoGebra help us?" ICM Satellite Conference Congreso llevado a cabo en Niteroi, Brasil.

Entradas.com (2019) "Técnicas y obras de improvisación teatral". Recuperado de: https://blogdeentradas.com/2019/09/27/tecnicas-y-obras-de-improvisacion-teatral/

Fernández, M. (6 de abril de 2020). “Tras la decisión de la UBA, ¿Cómo continuarán las clases en otras universidades?" Infobae. Recuperado de https:// www.infobae.com/educacion/2020/04/06/tras-la-decision-de-la-uba-como-continuara-las-clases-en-otras-universidades/

Magnani, E. (12 de abril de 2020) "Los riesgos de Zoom y las ventajas de Jitsi". Página 12. Recuperado de: https://www.pagina12.com.ar/258659-losriesgos-de-zoom-y-las-ventajas-de-jitsi

Universidad Nacional de La Plata (2020). "Comunicado: La UNLP ratifica la vigencia del calendario académico". Recuperado de https://unlp.edu.ar/coronavirus/comunicado-la-unlp-ratifica-la-vigencia-del-calendario-academico-17703 